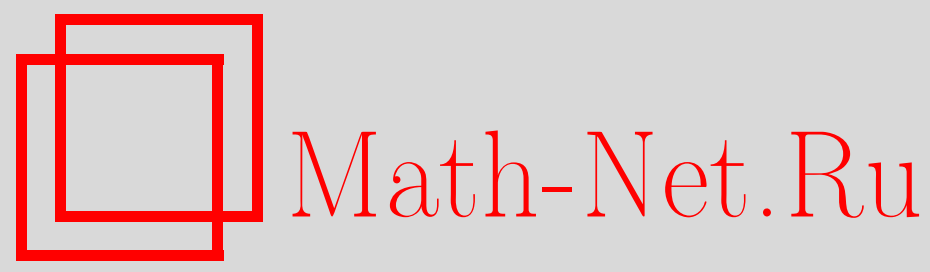

А. Н. Черепов, Оценки сложности приближения непрерывных функций некоторых классов детерминированными функциями с задержкой, Дискрет. матем., 2008, том 20, выпуск 4, 147-156

DOI: https://doi.org/10.4213/dm1034

Использование Общероссийского математического портала Math-Net.Ru подразумевает, что вы прочитали и согласны с пользовательским соглашением http://www .mathnet.ru/rus/agreement

Параметры загрузки:

IP : 54.197 .130 .99

26 апреля 2023 г., 16:56:20 


\title{
Оценки сложности приближения непрерывных функций некоторых классов детерминированными функциями с задержкой
}

\author{
() 2008 г. $\quad$ А. Н. Черепов
}

\begin{abstract}
Рассматриваются детерминированные функции с задержкой, являющиеся обобщением детерминированных функций. Вводится понятие сложности $\varepsilon$-приближения непрерывной действительной функции функцией с задержкой. Для ряда классов непрерывных функций, для которых известны оценки числа элементов $2 \varepsilon$-различимого множества функций, получены верхние и нижние оценки сложности.
\end{abstract}

\section{1. Введение}

Задача приближения функций является одной из классических в математике. Эта задача часто ставится следующим образом [2]. Пусть $(B,\|\cdot\|)-$ банахово пространство, а $H$ и $A-$ его подмножества, такие, что для любых $x \in H$ и $\varepsilon>0$ найдется $y \in A$, удовлетворяющий неравенству $\|x-y\|<\varepsilon$. На множестве $A$ вводится функционал $L$, сопоставляющий каждому $y \in A$ число $L(y)$, называемое сложностью элемента $y$. Под сложностью $\varepsilon$-приближения элемента $x$ понимается величина

$$
L(x, \varepsilon)=\inf \{L(y): y \in A,\|x-y\|<\varepsilon\},
$$

а сложностью приближения класса $H-$ число

$$
L(H, \varepsilon)=\sup \{L(x, \varepsilon): x \in H\} .
$$

В качестве $H$ чаще всего берется множество $C\left(I^{n}\right)$ всех непрерывных функций, определенных на $n$-мерном единичном кубе $I^{n}$ и принимающих значения из отрезка $[0,1]$. Норма $\|f\|$ функции $f \in C\left(I^{n}\right)$ определяется равенством

$$
\|f\|=\max \left\{|f(x)|: x \in I^{n}\right\} .
$$

В теории приближений, как правило, множество $A$ совпадает с классом всех полиномов (или рациональных функций), под сложностью понимается степень полинома (или рациональной функции), реализующего эту функцию.

Задача об оценке сложности реализации непрерывных функций и классов таких функций, была поставлена А. Н. Колмогоровым в начале 60-х годов XX века. При этом в качестве множества приближающих функций $A$ бралось множество дискретных функций, близких к автоматным [5]. Несколько иной подход был рассмотрен С. Б. Гашковым (см. 
[2]), множество $A$ при этом совпадает с множеством функций, реализуемых схемами в непрерывных базисах. В предлагаемой работе в качестве множества приближающих функций рассматривается класс дискретных детерминированных функций с задержкой. Этот класс появляется при естественном обобщении понятия детерминированной функции. В этом смысле задача приближения детерминированными функциями с задержкой является более близкой к колмогоровской постановке, чем подход, реализованный С. Б. Гашковым. Первые результаты о приближении непрерывных функций функциями с задержкой были получены Н. Ф. Тюленевым [6, 7]. В предлагаемой статье приводится подробное изложение результатов работ [8-11].

\section{2. Детерминированные функции с задержкой и их свойства}

Рассмотрим множество всех бесконечных двоичных последовательностей $E$. Множество всех функций вида $f: E^{n} \rightarrow E$ обозначим $P$. Предположим, что $a_{1}, \ldots, a_{n}-$ последовательности из $E$, а $\tilde{a}=\left(a_{1}, \ldots, a_{n}\right)$ - набор таких последовательностей. Пусть $a_{1}\left|k, \ldots, a_{n}\right| k-$ первые $k$ членов последовательностей $a_{1}, \ldots, a_{n}$ соответственно, тогда $\tilde{a} \mid k=\left(a_{1}\left|k, \ldots, a_{n}\right| k\right)$.

Назовем функцию $f$ детерминированной, если для всех $k=1,2, \ldots$ выполнено соотношение

$$
\forall \tilde{a}, \tilde{b}: \tilde{a}|k=\tilde{b}| k \rightarrow f(\tilde{a})|k=f(\tilde{b})| k
$$

Класс всех детерминированных функций обозначим $P_{d}$, все остальные функции из $P$ будем называть недетерминированными.

Определение 1 ([6]). Говорим, что функция $f$ является детерминированной функцией с задержкой $\tau$, где $\tau-$ произвольное неотрицательное целое число, если для любого $k=1,2, \ldots$ и любых $\tilde{a}, \tilde{b}$

$$
\tilde{a}|k+\tau=\tilde{b}| k+\tau \Longrightarrow f(\tilde{a})|k=f(\tilde{b})| k .
$$

Множество всех функций с задержкой $\tau$ обозначим $P_{d}^{\tau}$. Заметим, что $P_{d}^{0}=P_{d}$.

Множество $P_{d}^{\tau}$ можно определить и следующим образом.

Определение 2. Говорим, что функция $f$ является детерминированной функцией с задержкой $\tau$, где $\tau$ - произвольное неотрицательное целое число, если существует такая детерминированная функция $g$, что для любого $\tilde{a}$ и $b=g(\tilde{a}), b=b(1) b(2) b(3) \ldots$ значение функции $f$ на наборе $\tilde{a}$ равно $f(\tilde{a})=b(\tau+1) b(\tau+2) b(\tau+3) \ldots$

Предложение 1 ([9, 10]). Определения 1 и 2 эквивалентнь.

Из определения 2 следует, что функцию $n$ аргументов из множества $P_{d}^{\tau}$ можно трактовать так: берется дискретное детерминированное устройство с $n$ входами, преобразующее бесконечные входные последовательности из нулей и единиц в такую же выходную последовательность и рассматривается выход этого устройства не с первого момента времени, а с момента времени $\tau+1$. Осуществляемое преобразование и считается функцией множества $P_{d}^{\tau}$. Таким образом, функции с задержкой являются естественным обобщение детерминированных функций. 


\section{3. Приближение непрерывных функций действительными функциями с задержкой}

Перейдем к функциям вида $f: I^{n} \rightarrow[0,1]$. При этом будем считать, что числа отрезка $[0,1]$ представлены в двоичной системе счисления и для чисел, допускающих два представления, выберется представление с бесконечным хвостом из нулей. Для числа 1 выберем представление $1=0,111 \ldots$

Определение 3. Пусть $\alpha=0, \alpha_{1} \alpha_{2} \ldots, \beta=0, \beta_{1} \beta_{2} \ldots$ - действительные числа отрезка $[0,1]$ и $k$ - натуральное число. Тогда будем считать, что $\alpha|k=\beta| k$, если у чисел $\alpha, \beta$ совпадают первые $k$ двоичных разряда. Для точек $\tilde{a}, \tilde{b} \in[0,1]^{n}$ справедливо равенство $\tilde{a}|k=\tilde{b}| k$, если для каждой координаты выполнено равенство $a_{i}\left|k=b_{i}\right| k$. Действительную функцию $f$ назовем детерминированной, если для любого $k=1,2, \ldots$ из $\tilde{a}|k=\tilde{b}| k$ следует, что $f(\tilde{a})|k=f(\tilde{b})| k$. Класс всех детерминированных функций обозначим $D_{d}$. В множество $D_{d}^{\tau}$ действительных детерминированных функций с задержкой $\tau$ включим все функции, удовлетворяющие условию: для любого $k=1,2, \ldots$ из равенства $\tilde{a}|k+\tau=\tilde{b}| k+\tau$ следует, что $f(\tilde{a})|k=f(\tilde{b})| k$.

Класс $D_{d}^{\infty}$ определим как объединение всех множеств $D_{d}^{\tau}$.

Пусть $\tilde{a}=\left(a_{1}, a_{2}, \ldots, a_{n}\right) \in E^{n}, b \in E$ и $\tilde{a}, b$ не заканчиваются бесконечными последовательностями из единиц. Пусть $\tilde{\alpha}=\left(0, a_{1}^{1} a_{2}^{1} \ldots ; 0, a_{1}^{2} a_{2}^{2} \ldots ; \ldots ; 0, a_{1}^{n} a_{2}^{n} \ldots\right)$ и $\beta=0, b_{1} b_{2} \ldots$, где двоичные разряды чисел совпадают с значениями последовательностей $\tilde{a}=\left(a_{1}, a_{2}, \ldots, a_{n}\right), b$. Дискретную функцию $f: E^{n} \rightarrow E$ и действительную функцию $d: I^{n} \rightarrow[0,1]$ назовем соответствующими друг другу, если $d(\tilde{\alpha})=\beta$ для любых таких $\tilde{a}, b$. Множества $D_{d}^{\tau}$ и $D_{d}^{\infty}$ могут быть построены по соответствующим им классам дискретных функций при потере части информации о значениях дискретных функций на последовательностях вида $a(1) a(2) \ldots a(i) 0111 \ldots$

Определение 4. Пусть $\varepsilon>0$, будем говорить, что функция $d(\tilde{x}) \varepsilon$-равна функции $f(\tilde{x})$ на единичном кубе $I^{n}$, если при любом $\tilde{x} \in I^{n}$ справедливо неравенство $|f(\tilde{x})-d(\tilde{x})|<\varepsilon$. Будем также говорить, что $d(\tilde{x}) \quad \varepsilon$-приближает $f(\tilde{x})$.

Пусть $C\left(I^{n}\right)$ - множество непрерывных на единичном кубе $I^{n}$ функций, принимающих значения из отрезка $[0,1]$. Оказывается, что не всякую функцию множества $C\left(I^{n}\right)$ можно $\varepsilon$-приблизить детерминированными функциями класса $D_{d}$, но функций класса $D_{d}^{\infty}$ уже достаточно для решения этой задачи.

Теорема $1([6,9,10])$. Для любого $\varepsilon$ и любой функиии $f(\tilde{x}) \in C\left(I^{n}\right)$ существует нату-

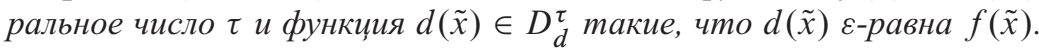

Определение 5. Под константной функцией с задержкой $\tau$ понимается функция множества $D_{d}^{\tau}$, такая, что соответствующая ей функция $P_{d}^{\tau}$ на выходе после задержки $\tau$ выдает последовательность значений, не зависящую от значений входных сигналов, поступающих после задержки. Назовем функцию $f(\tilde{x})$ ограниченной $m$ разрядами, если существует такое $m$, что при любом $\tilde{x} \in I^{n}$ справедливо равенство $f(\tilde{x})=0, a_{1} a_{2} \ldots a_{m} 00 \ldots$

Предложение 2. Для любого $\varepsilon=2^{-k}$ и любой функиии $f(\tilde{x}) \in C\left(I^{n}\right)$ существует число $\tau$ и константная функиия $d(\tilde{x}) \in D_{d}^{\tau}$, ограниченная $k+1$ разрядом, такие, что $d(\tilde{x}) \varepsilon-$ равна $f(\tilde{x})$. При этом дискретная функиия $r(\tilde{x})$, соответствующая функиии $d(\tilde{x})$, может быть получена с помощью задержки $\tau$ из некоторой ограниченно детерминированной функиии. 
Доказательство. Возьмем $\varepsilon_{1}=2^{-k-1}$. Пусть $g \in D_{d}^{\tau}-$ функция, $\varepsilon_{1}$-приближающая $f$. Заметим, что если у функций $f$ и $g$ при любом $\tilde{x}$ совпадают первые $k+1$ разряда, то $|d(\tilde{x})-g(\tilde{x})|<\varepsilon_{1}$ при всех $x$. Для произвольного $\tilde{x}$ определим значение $y=d(\tilde{x})$, полагая, что первые $k+1$ разряда $y$ совпадают с соответствующими первыми разрядами значения $g(\tilde{x})$, а остальные разряды равны 0 . Тогда при любом $\tilde{x}$

$$
\begin{aligned}
\mid(f(\tilde{x})-d(\tilde{x}) \mid & =|(f(\tilde{x})-g(\tilde{x}))+(g(\tilde{x})-d(\tilde{x}))| \\
& \leqslant|f(\tilde{x})-g(\tilde{x})|+|g(\tilde{x})-d(\tilde{x})|<\varepsilon_{1}+\varepsilon_{1}=\varepsilon,
\end{aligned}
$$

то есть функция $d \varepsilon$-приближает $f$ и принадлежит множеству $D_{d}^{\tau}$.

Рассмотрим дискретную функцию $r(\tilde{x})$, соответствующую $d(\tilde{x})$. Достаточно показать, что дискретная функция, соответствующая константной функции, ограниченной $k+1$ разрядом, может быть получена из некоторой ограниченно-детерминированной функции с помощью задержки $\tau$. Такую функцию можно описать с помощью двоичного дерева следующим образом. Ребрам двоичного дерева, соответствующего задержке, припишем произвольные выходные значения. В каждую висячую вершину поместим ограниченно-детерминированную функцию, реализующую двоичную последовательность $a_{1} a_{2} \ldots a_{k+1} 00 \ldots$, первые элементы которой совпадают с первыми $k+1$ разрядами значения функции $d(\tilde{x})$, а остальные значения равны 0 .

В дальнейшем дискретную функцию $r(\tilde{x})$ будем называть $(\tau, m)$-реализацией действительной функции $d(\tilde{x})$, если она соответствует константной функции $r(\tilde{x})$ с задержкой $\tau$, ограниченной $m$ разрядами.

Предложение 3. Число различных константных функций $n$ переменных $c$ задержкой $\tau$, ограниченных $m$ разрядами, равно $2^{m 2^{n \tau}}$.

Доказательство. Подсчитаем число дискретных функций с задержкой $\tau$, соответствующих константным функциям $n$ переменных, ограниченных $m$ разрядами. Для произвольной константной функции $d(\tilde{x})$ рассмотрим ее $(\tau, m)$ реализацию. Задержке $\tau$ будет соответствовать двоичное дерево высоты $\tau$, имеющее $2^{n \tau}$ висячие вершины. В каждой висячей вершине расположена ограниченно-детерминированная функция, реализующая двоичную выходную последовательность $a_{1} a_{2} \ldots a_{m} 00 \ldots$ Различных таких функций будет столько же, сколько существует двоичных наборов длины $m$, то есть $2^{m}$. Следовательно, различных константных функций с задержкой $\tau$, ограниченных $m$ разрядами будет $2^{m 2^{n \tau}}$.

\section{4. Оценки сложности приближения для некоторых классов функций}

Определение 6. Для любой непрерывной функции $f \in C\left(I^{n}\right)$ и любого $\varepsilon<1$ назовем сложностью реализации в классе детерминированных функций с задержкой $\tau$ наименьшее $\tau$ такое, что существует функция $d(\tilde{x})$ класса $D_{d}^{\tau} \quad \varepsilon$-равная $f$. Сложность реализации функции $f$ будем обозначать $L(f, \varepsilon)$. Пусть задан подкласс $H$ класса непрерывных на множестве $I^{n}$ функций, принимающих значения из отрезка $[0,1]$. Положим

$$
L(H, \varepsilon)=\sup (L(f, \varepsilon): f \in H) .
$$


Укажем оценки величины $L(H, \varepsilon)$ для некоторых известных классов непрерывных функций, для которых найдены оценки числа $2 \varepsilon$-различимых функций (см. $[1,4,5])$.

Введем следующие обозначения [3]. Будем считать, что

$$
f(n)=O(g(n)) \Longleftrightarrow|f(n)| \leqslant C|g(n)|
$$

для некоторой константы $C>0$ и всех $n$. Кроме того,

$$
f(n) \preceq g(n) \Longleftrightarrow \varlimsup_{n \rightarrow \infty} \frac{f(n)}{g(n)}<\infty .
$$

Если $f(n) \preceq g(n)$ и $f(n) \preceq g(n)$, то $f(n) \asymp g(n)$.

\section{1. Класс функций, удовлетворяющих условию Гельдера}

Напомним, что функция $f(\tilde{x})$ удовлетворяет условию Гельдера с показателем $\alpha$ и константой $L$, если для любых $\tilde{x}, \tilde{y}$

$$
|f(\tilde{x})-f(\tilde{y})| \leqslant L\|\tilde{x}-\tilde{y}\|^{\alpha},
$$

где в качестве нормы рассматривается максимум модуля разности координат. В дальнейшем будем полагать $L=1$, что не ограничивает общности рассмотрения. Обозначим класс функций от не более чем $n$ аргументов, удовлетворяющих условию Гельдера с показателем $\alpha$ и константой $L=1$, через $G_{n}(\alpha)$.

Предложение 4. Справедливо неравенство

$$
L\left(G_{1}(\alpha), \varepsilon\right) \leqslant \frac{1}{\alpha} \log \frac{1}{\varepsilon}+\frac{1}{\alpha} .
$$

Здесь и в дальнейшем логарифм берется по основанию 2.

Доказательство. Заметим, что приращение функции $f \in G_{1}(\alpha)$ на отрезке длины $\delta$ не превосходит $\delta^{\alpha}$. Рассмотрим разбиение отрезка $[0,1]$ с шагом $h=2^{-\tau}$ точками $0,2^{-\tau}, \ldots$, $i 2^{-\tau}, \ldots, 1$ при

$$
\tau=\left\lceil\frac{1}{\alpha} \log \frac{1}{\varepsilon}\right\rceil+\frac{1}{\alpha},
$$

где $\lceil a\rceil$ означает целую часть числа $a$ с избытком. Такое разбиение будем в дальнейшем называть решеткой $I_{h}$. Тогда длина каждого из отрезков решетки не превосходит $2^{-\tau} \leqslant 2^{-(1 / \alpha) \log (1 / \varepsilon)-1 / \alpha}$. Приращение функции $f$ на каждом таком отрезке не более $2^{-\tau \alpha} \leqslant 2^{-\log (1 / \varepsilon)-1}=\varepsilon / 2$. Построим функцию $d(x)$ с задержкой $\tau, \varepsilon$-равную функции $f(x)$. Для этого рассмотрим дискретную функцию с задержкой $\tau$, устроенную следующим образом. В висячую вершину, которая соответствует последовательности входных двоичных символов $a(1), \ldots, a(\tau)$, поместим детерминированную функцию, выдающую последовательность, совпадающую с первыми $k+1$ двоичными разрядами числа $f(a)$, $a=0, a(1) \ldots a(\tau) 00 \ldots$, а затем нули. Число $k$ выберем равным $k=\lceil\log (1 / \varepsilon)\rceil$. Тогда при $a=0, a(1) a(2) \ldots a(\tau) 00 \ldots$ справедливо неравенство

$$
|f(a)-d(a)| \leqslant 2^{-(k+1)}<2^{-\log (1 / \varepsilon)-1}=\varepsilon / 2 .
$$

Поскольку изменение функции $f$ на каждом из отрезков решетки $I_{h}$ не превосходит $\varepsilon / 2$, в любой точке $x$ отрезка решетки $|f(x)-d(x)|<\varepsilon$. Отсюда следует оценка

$$
L\left(G_{1}(\alpha), \varepsilon\right) \leqslant \frac{1}{\alpha} \log \frac{1}{\varepsilon}+\frac{1}{\alpha} \leqslant C \frac{1}{\alpha} \log \frac{1}{\varepsilon},
$$

где $C-$ некоторая положительная константа, не зависящая от $\alpha$. 
Следствие 1. Для класса непрерывных функиий $G_{1}(1)$, удовлетворяющих условию Липший, справедлива оченка

$$
L\left(G_{1}(1), \varepsilon\right) \leqslant \log \frac{1}{\varepsilon}+1
$$

или

$$
L\left(G_{1}(1), \varepsilon\right)=O\left(\log \frac{1}{\varepsilon}\right) .
$$

Определение 7 ([1,4]). Множество функций $M$ называется $\varepsilon$-различимым на множестве $G$, если для любых функций $f_{1}, f_{2}$ из этого множества справедливо неравенство $\sup \left|f_{1}-f_{2}\right|>\varepsilon$, где супремум ищется на множестве $G$.

Предложение 5 ([1]). В множестве функиий $G_{1}(1)$ с дополнительным условием $f(0)=0$ существует $2 \varepsilon$-различимое подмножество функиий. Мощчность этого подмножества имеет порядок $2^{C / \varepsilon}$.

Используя это утверждение, можно получить нижнюю оценку для $L\left(G_{1}(1), \varepsilon\right)$.

Предложение 6. Справедливо соотношение

$$
\left.L\left(G_{1}(1), \varepsilon\right) \asymp \log (1 / \varepsilon)\right) .
$$

Доказательство. Поскольку верхняя оценка уже получена, достаточно получить нижнюю оценку. Пусть $k=\lceil\log (1 / \varepsilon)\rceil$. Согласно предложению 2, любую функцию класса $G_{1}(1)$ можно приблизить некоторой функцией, имеющей $(\tau, k+2)$-реализацию. Мощность множества $(\tau, k+2)$-реализаций не может быть меньше мощности $2 \varepsilon$-различимого подмножества функций из $G_{1}(1)$, поэтому, учитывая предложение 3 , получаем, что $2^{(k+2) 2^{\tau}} \succeq 2^{C / \varepsilon}$ или $(k+2) 2^{\tau} \succeq C / \varepsilon$. Поскольку $k=\lceil\log (1 / \varepsilon)\rceil$, справедливо соотношение

$$
\tau \succeq \log C+\log (1 / \varepsilon)-\log (\log (1 / \varepsilon)+2)
$$

так, что $\tau \asymp \log (1 / \varepsilon)$.

\section{2. Класс $F_{r}$}

Класс $F_{r}$ определяется как класс непрерывных на отрезке $[0,1]$ функций, имеющих $r$ производных. Функции $f \in F_{r}$ и их производные удовлетворяют неравенствам

$$
\max \left|f^{(i)}(x)\right| \leqslant 1, \quad i=1, \ldots, r,
$$

где максимум берется на отрезке $[0,1]$. При этом выполнены условия

$$
f^{(i)}(0)=0, \quad i=1, \ldots, r-1 .
$$

Оценим величину $L\left(F_{r}, \varepsilon\right)$. Для этого воспользуемся тем, что функции класса $F_{r}$ удовлетворяют условию Липшица с константой 1, и следствием 1 из предложения 4.

Предложение 7. Для функиий класса $F_{r}$ справедлива верхняя оценка

$$
L\left(F_{r}, \varepsilon\right) \leqslant \log \frac{1}{\varepsilon}+1 .
$$

Предложение 8 ([1, 4,5]). В классе $F_{r}$ существует $2 \varepsilon$-различимое множество, содержащее порядка $2^{C(1 / \varepsilon)^{1 / r}}$ функиий. 
Используя это предложение, получим следующий утверждение.

Предложение 9. Для функций класса $F_{r}$ справедлива нижняя оценка

$$
L\left(F_{r}, \varepsilon\right) \succeq \log \frac{1}{\varepsilon} .
$$

Доказательство. Для любой функции класса $F_{r}$ имеется $(\tau, k+2)$-реализация, где $k=\lceil\log (1 / \varepsilon)\rceil$. Число таких реализаций не может быть меньше числа $2 \varepsilon$-различимых функций класса $F_{r}$, поэтому число $2^{(k+2) 2^{\tau}}$ не может быть меньше числа $2^{C(1 / \varepsilon)^{1 / r}}$. Поэтому

$$
(k+2) 2^{\tau} \succeq C(1 / \varepsilon)^{1 / r}
$$

Отсюда следует, что

$$
\tau \succeq \log C+\frac{1}{r} \log \frac{1}{\varepsilon}-\log \left(\log \frac{1}{\varepsilon}+2\right) .
$$

Следовательно, $\tau \succeq \log (1 / \varepsilon)$.

\section{3. Класс функций от $n$ аргументов, удовлетворяющих условию Гельдера}

Пусть $E^{n}$ есть $n$-мерное евклидово пространство и $I^{n}$ есть $n$-мерный замкнутый куб, задаваемый неравенствами $0 \leqslant x_{i} \leqslant 1, i=1, \ldots, n$. Рассмотрим множество $F_{r} G_{n}(\alpha)$ действительных функций $n$ переменных, определенных на $I^{n}$, все частные производные которых порядка не выше $r$ удовлетворяют условию Гельдера с показателем $0<\alpha \leqslant 1$ и константой $L=1$ и таких, что при $\sum_{i=1}^{n} k_{i} \leqslant r$

$$
\left|\frac{\delta^{k_{1}+k_{2}+\ldots+k_{n}}}{\delta_{x_{1}}^{k_{1}} \delta_{x_{2}}^{k_{2}} \ldots \delta_{x_{n}}^{k_{n}}} f(0, \ldots, 0)\right| \leqslant 1 .
$$

Предложение 10. Справедлива оцченка

$$
L\left(F_{r} G_{n}(\alpha), \varepsilon\right) \geqslant \frac{1}{\alpha} \log \frac{1}{\varepsilon}+\frac{1}{\alpha} .
$$

Доказательство. Рассмотрим решетку $I_{h}$, где $h=2^{-\tau}$. В $n$-мерном кубе $I^{n}$ введем решетку $I_{h}^{n}$ как декартову степень решетки $I_{h}$. Пусть $\tilde{a}=\left(a_{1}, a_{2}, \ldots, a_{n}\right)-$ произвольная точка решетки $I_{h}^{n}$. Интервалом $I_{h}(\tilde{a})$ решетки $I_{h}^{n}$ назовем множество точек $\tilde{x}=\left(x_{1}, x_{2}, \ldots, x_{n}\right)$ куба $I_{n}$ таких, что $a_{i} \leqslant x_{i}<h$ при $i=1,2, \ldots$ На каждом из интервалов, определяемых точками разбиения, изменение любой функции $f$ из множества $F_{r} G_{n}(\alpha)$ не превосходит $2^{-\tau \alpha}$. Поэтому при

$$
\tau \geqslant \frac{1}{\alpha} \log \frac{1}{\varepsilon}+\frac{1}{\alpha}
$$

это изменение не больше $\varepsilon / 2$.

Таким образом, при таком выборе $\tau$ достаточно задать значение функции $f$ в одной из точек интервала $I_{h}(\tilde{a})$ разбиения куба $I^{n}$ с точностью $\varepsilon / 2$. В качестве такой точки можно выбрать, например, точку $\tilde{a}$. При $k=\lceil\log (1 / \varepsilon)\rceil$ соответствующая $(\tau, k+2)$ реализация строится аналогично $(\tau, k+2)$-реализации из доказательства предложения 2 : 
берется двоичное дерево высоты $\tau$, каждой висячей вершине которого соответствует точка $\tilde{a}$ разбиения $I_{h}^{n}$ куба $I^{n}$ (заметим, что число висячих вершин равно $2^{n \tau}$ ). В каждую висячую вершину дерева поместим детерминированную функцию, реализующую константу, равную значению приближаемой функции в этой точке с точностью $\varepsilon / 2$. Предложение доказано.

Предложение 11 ([1,5,11]). B классе $F_{r} G_{n}(\alpha)$ существует $2 \varepsilon$-различимое множество, содержащее порядка $2^{C /(\varepsilon)^{(n / s)}}$ функичй, где $s=r+\alpha$.

Из этого предложения получаем следующее утверждение.

Предложение 12. Справедлива оценка

$$
L\left(F_{r} G_{n}(\alpha), \varepsilon\right) \succeq \log \frac{1}{\varepsilon} .
$$

Доказательство. Подсчитаем число возможных $(\tau, k+2)$-реализаций функций множества $F_{r} G_{n}(\alpha)$. Оно не превосходит $2^{(k+2) 2^{n \tau}}$, где $k=\lceil\log (1 / \varepsilon)\rceil$. Каждую функцию класса $F_{r} G_{n}(\alpha)$ можно приблизить с помощью некоторой $(\tau, k+2)$-реализации. Число возможных реализаций не может быть меньше числа функций $2 \varepsilon$-различимого множества, поэтому

$$
2^{(k+2) 2^{n \tau}} \succeq 2^{C /(\varepsilon)^{n / s}}
$$

или

$$
\begin{gathered}
(k+2) 2^{n \tau} \succeq C /(\varepsilon)^{n / s}, \\
n \tau \succeq \frac{n}{s} \log \frac{1}{\varepsilon}+\log C-\log \left(\log \frac{1}{\varepsilon}+2\right) .
\end{gathered}
$$

Отсюда,

$$
L\left(F_{r} G_{n}(\alpha), \varepsilon\right) \succeq \log \frac{1}{\varepsilon} .
$$

Следствие 2. При $0<\alpha \leqslant 1$ справедлива оцеенка

$$
L\left(G_{1}(\alpha), \varepsilon\right) \succeq \log \frac{1}{\varepsilon} .
$$

Эта оценка получается из предыдущей при $r=0$ и $n=1$.

\section{4. Множество $F_{A}$ аналитических функций}

Множество $F_{A}$ определяется как класс аналитических в некоторой области функций, производные которых удовлетворяют неравенствам

$$
\max \left|\frac{f^{(i)}(x)}{i !}\right| \leqslant \frac{1}{2^{i}}, \quad i=1,2, \ldots
$$

Поскольку функции этого класса удовлетворяют условию Липшица с константой 1 , справедливо следующее утверждение.

Предложение 13. Для функций класса $F_{A}$ верна верхняя оценка

$$
L\left(F_{A}, \varepsilon\right) \leqslant \log \frac{1}{\varepsilon}+1 .
$$


Таблица 1.

\begin{tabular}{ccc}
\hline Класс & Нижняя оценка & Верхняя оценка \\
\hline$G_{1}(1)$ & $\log \frac{1}{\varepsilon}$ & $\log \frac{1}{\varepsilon}+1$ \\
$G_{1}(\alpha)$ & $\log \frac{1}{\varepsilon}$ & $\frac{1}{\alpha} \log \frac{1}{\varepsilon}+1$ \\
$F_{r} G_{n}(\alpha)$ & $\log \frac{1}{\varepsilon}$ & $\frac{1}{\alpha} \log \frac{1}{\varepsilon}+\frac{1}{\alpha}$ \\
$F_{r}$ & $\log \frac{1}{\varepsilon}$ & $\log \frac{1}{\varepsilon}+1$ \\
$F_{A}$ & $\log \left(\log \frac{1}{\varepsilon}\right)$ & $\log \frac{1}{\varepsilon}+1$ \\
\hline
\end{tabular}

Предложение 14 ([1,5]). В компакте $F_{A}$ существует $2 \varepsilon$-различимое множество функиций, содержащчее порядка $2^{C \log ^{2}(1 / \varepsilon)}$ функичй.

Сравнивая эту величину с числом возможных $(\tau, k+2)$-реализаций, получаем следующее утверждение.

Предложение 15. Для функиий класса $F_{A}$ справедлива нижняя оценка

$$
L\left(F_{A}, \varepsilon\right) \succeq \log (\log (1 / \varepsilon)) .
$$

Доказательство. Возьмем $k=\lceil\log (1 / \varepsilon)\rceil$ и сравним число $(\tau, k+2)$-реализаций с порядком числа функций в $2 \varepsilon$-различимом множестве. Получаем неравенство

$$
2^{(k+2) 2^{\tau}} \succeq 2^{C \log ^{2}(1 / \varepsilon)},
$$

или

$$
\begin{gathered}
(k+2) 2^{\tau} \succeq C \log ^{2}(1 / \varepsilon), \\
\tau \succeq \log C+2 \log (\log (1 / \varepsilon))-\log (k+2) .
\end{gathered}
$$

Отсюда получаем, что

$$
L\left(F_{A}, \varepsilon\right) \succeq \log (\log (1 / \varepsilon))
$$

В Табл. 1 приводятся оценки сложности реализаций детерминированными функциями с задержкой для рассмотренных классов. В этой таблице нижние оценки являются оценками по порядку.

Сопоставим полученные результаты с результатами работы [5], где непрерывные функции приближались многозвенными автоматами. Для классов $G_{1}(1)$ и $G_{1}(\alpha)$ верхние и нижние оценки в [5] совпадают, как и в нашем случае. Для класса $F_{r}$ в [5] получена верхняя оценка, совпадающая с нижней. Для класса $F_{A}$ в [5] верхняя оценка не совпадает с нижней. Класс функций $F_{r} G_{n}(\alpha)$ в [5] не рассматривался.

В заключение автор выражает свою признательность В. А. Буевичу за постановку задачи и существенную помощь при оформлении полученных результатов.

\section{Список литературы}

1. Витушкин А. Г., Оценка сложности задачи табулящуии. Физматгиз, Москва, 1959. 
2. Гашков С. Б., О сложности приближенной реализации непрерывных функций схемами и формулами в полиномиальных и некоторых других базисах. Матем. вопросы кибернетики (1994) 5, 144-207.

3. Грехем Р., Кнут Д., Паташник О., Конкретная математика. Мир, Москва, 1998.

4. Колмогоров А. Н., Тихомиров В. М., $\varepsilon$-энтропия и $\varepsilon$-емкость множеств в функциональных пространствах. Успехи матем. наук (1959) 14, №2, 3-86.

5. Офман Ю. П., О приближенной реализации непрерывных функций на автоматах. Доклады $A H$ СССР (1963) 152, №4, 823-825.

6. Тюленев Н. Ф., Приближение непрерывных функций дискретными. В сб.: Сборник трудов семинара по дискретной математике и ее приложениям. Мех.-мат. ф-т МГУ, Москва, 1997, c. $148-151$.

7. Тюленев Н. Ф., О приближении непрерывных функций дискретными. В сб.: Конструкции в алгебре и логике. Тверской госунив., Тверь, 1990, с. 110-116.

8. Черепов И. А., О приближении непрерывных функций детерминированными функциями с задержкой. В сб.: Сборник трудов семинара по дискретной математике и ее приложениям. Мех.-мат. ф-т МГУ, Москва, 2004, с. 163-166.

9. Черепов А. Н., Черепов И. А., О классификации недетерминированных функций. В сб.: Сборник трудов семинара по дискретной математике и ее приложениям. Мех.-мат. ф-т МГУ, Москва, 2004, c. 160-163.

10. Черепов А. Н., О сложности приближения непрерывных функций недетерминированными функциями с задержкой. В сб.: Материаль IX Международной конференции «Интеллектуальные системы и компьютерные науки», 1 (2). Мех.-мат. ф-т МГУ, Москва, 2006, с. 307-310.

11. Черепов А. Н., Оценки сложности приближения непрерывных функций некоторых классов детерминированными функциями с задержкой. В сб.: Материалы XVI Международной школьсеминара «Синтез и сложность управляющих систем». Мех.-мат. ф-т МГУ, Москва, 2006, c. $118-122$.

Статья поступила 28.11.2007. 